\title{
KMT2D and TP53 mutation status improve the prognostic value of the International Prognostic Index (IPI) stratification in ENKTL patients
}

\author{
Y. GAO*, Y. LI*, G. MA, G. ZHAO, H. LIU \\ Department of Hematology, Fourth Hospital of Hebei Medical University, Shijiazhuang, Hebei, China \\ *Correspondence: ydsygao@sina.com; 419136407@qq.com
}

Received March 07, 2019 / Accepted August 11, 2019

\begin{abstract}
Extranodal NK/T-cell lymphoma nasal type (ENKTL) is a subtype of T cell lymphoma with poor prognosis. In this study, we designed a new prognostic model specifically for ENKTL to improve the risk stratification. In 29 ENKTL patients, we screened mutations in 9 ENKTL-associated genes using next-generation sequencing (NGS). We have found that mutated KMT2D was associated with the inferior overall survival (OS) and progression free survival (PFS) and KMT2D or TP53 mutations were associated with a higher mortality rate. Moreover, the difference in PFS among different stratifications was not significant using the International Prognostic Index (IPI) alone but was significant after the mutation status of KMT2D and TP53 were incorporated into the IPI model, forming a harmonious risk stratification reflecting the clinical features and genetic information of ENKTL. In summary, we demonstrate that the prognostic value of the IPI system can be enhanced by integrating the status of genetic mutations.
\end{abstract}

Key words: ENKTL, IPI, risk stratification, NGS, gene profile, KMT2D

Extranodal NK/T cell lymphoma (ENKTL) is a subtype of non-Hodgkin's lymphoma (NHL), characterized by a cytotoxic phenotype and is associated with Epstein-Barr virus (EBV) infection [1]. ENKTL is an aggressive disease with an incidence of about $5-18 \%$ of NHL [2] and most commonly occurs in East Asia and Latin America [3]. Although the survival time of patients has been improved by chemoradiotherapy and autologous stem cell transplantation, the risk of relapse and drug resistance is still high [4]. At present, optimal treatment strategies and prognosis for patients with ENKTL have not been fully defined [5].

The International Non-Hodgkin's Lymphoma Prognostic Factors Project proposed a prognosis model based on clinical characteristics, including age, tumor stage, serum lactate dehydrogenase (LDH) concentration, performance status, and the number of extranodal disease sites for NHL [6]. Although the above international prognostic index (IPI) has been widely used for both predicting prognosis and selecting therapeutic options in patients with aggressive NHL, it has not been widely used in ENKTL because of the inhomogeneous distribution found for this disease. More than half of ENKTL patients were stratified at low risk according to the IPI classification [7], leading to inaccurate prognoses. Moreover, clinical features do not fully reflect the biological characteristics of ENKTL. Therefore, it is urgent to identify new prognostic indicators to further improve the IPI classification to better stratify and guide the treatment of ENKTL patients.

Recently, many studies have shown that gene mutations are associated with the classification and prognostic evaluation of lymphoma [8-10]. For example, ARID1A and EP300 gene mutations detected by next-generation sequencing (NGS) improved IPI score accuracy and improved prognosis of follicular lymphoma [11]. NGS can simultaneously carry out sequence analysis of hundreds of thousands or millions of short DNA molecules, in high throughput formats with high sensitivity and relative quantification [12]. Moreover, a recent multicenter laboratory evaluation showed that NGS was highly consistent and repeatable [13]. However, the prognostic value of genetic mutations has not been entirely assessed in ENKTL populations. We retrospectively investigated a cohort of ENKTL patients by NGS and detected mutations in nine candidate genes highly associated with ENKTL pathogenesis and prognosis. We aimed to improve the risk stratification of ENKTL patients by integrating gene mutations into IPI classifications, providing more prognostic information for personalized therapeutic strategies. 


\section{Patients and methods}

Patients. We retrospectively reviewed a total of 29 patients with primary ENKTL at 4th Hospital of Hebei Medical University from August 2010 to November 2016. Review, analysis, and publication of the data from this study were approved by the ethics committee of Hebei Medical University. Informed consent for the collection of medical information was obtained from all patients. The data collected at diagnosis included patient age, gender, Ann Arbor stage [14], Eastern Cooperative Oncology Group performance status (ECOG PS), regional lymph node quantification, extranodal sites, B symptoms, complete blood count, serum $\mathrm{LDH}$, biochemical profile, bone marrow examination, and computed tomography scanning. The diagnosis and clinical stage of all patients were determined according to the 2008 WHO classification scheme of hematopoietic and lymphoid tissue tumors [15]. The 29 patients were all treated by at least 2 courses of asparaginase based chemotherapy (CT) alone or combined with field radiotherapy (RT) after diagnosis with ENKTL.

Next generation sequencing. In this study, we designed a small panel of ENKTL-related genes including ARID1A, KMT2D, TP53, MGA, STAT3, EP300, ASXL3, DDX3X and $S T A T 5 B$ for deep sequencing to investigate gene mutation status in our ENKTL patient cohort. Genomic DNA from paraffin-embedded tissue was isolated with QIAamp DNA FFPE kit (Qiagen, CA, USA) according to the manufacturer's instructions. Primers for the nine genes for NGS were custom-designed and synthesized by Yuanqi Bio-Pharmaceutical Co., Ltd. (Shanghai, China). The main steps of NGS were as follows: a genomic DNA (gDNA) library was prepared using the TruSeq Nano DNA Library Preparation Kit (Illumina, San Diego, CA, USA). The gDNA was sheared into fragments by sonication and ligated at both ends with indexed paired-end adaptors (Covaris, Woburn, MA, USA). Then the adapter-ligated gDNAs were purified and used as templates in a ligation-mediated PCR for 8 cycles to enrich target genes. Paired-end DNA was sequenced on a HiSeq X (Illumina, San Diego, CA, USA). Data analysis was performed using Illumina bcl2fastq software, version 2.15.

Risk stratification scoring system for ENKTL. IPI scores were calculated based on clinical features including age, tumor stage, $\mathrm{LDH}$ concentration, performance status, and the number of extranodal disease sites. Scores for our new classification were calculated by combining the IPI score with a factor based on KMT2D and TP53 mutation status. Details were as follows: wild-type was assigned ' 0 ', mutant was assigned ' 1 ', and the score = sum (IPI score $+K M T 2 D$ + TP53). We defined three categories to establish the new integrated system: low risk (0-1 point), intermediate risk (2-3 points) and high risk (4-6 points).

Statistical analysis. All data were analyzed using IBM SPSS statistics software (Version 20.0, SPSS Inc., USA). The treatment effect on overall survival (OS) rate and progres- sion-free survival (PFS) rate was determined by Kaplan Meier curves. $P<0.05$ was considered statistically significant.

\section{Results}

Clinical features of ENKTL patients. We recruited a total of 29 patients with primary ENKTL for this study at the Fourth Hospital of Hebei Medical University from August 2010 to February 2017. The median age was 46 years (range 25-73 years), with $24(82.8 \%)$ being male and $5(17.2 \%)$ female (Table 1). According to the Ann Arbor standard, 19 patients were in phases I/II and 10 patients were in phases III/ IV. We recorded abnormal clinical features including lactate dehydrogenase (LDH, threshold $250 \mathrm{U} / \mathrm{l}$ ), Ki67 (threshold $70 \%$ ), albumin (ALB, threshold $40 \mathrm{~g} / \mathrm{l}$ ) and absolute lymphocyte count (ALC, threshold $1.1 \times 10^{9} / 1$ ) as follows: increased $\mathrm{LDH}$ and $\mathrm{Ki} 67$ in $44.8 \%$ and $24.1 \%$ of patients, respectively; decreased ALB and ALC in $48.3 \%$ and $44.8 \%$ of patients, respectively. According to the IPI classification, we found 14, 8, 4 and 3 ENKTL patients with low, low-to-intermediate, intermediate-to-high and high risk, respectively. Seventeen patients received chemotherapy plus radiotherapy and 12 patients received chemotherapy alone. After treatment, 16 patients achieved a complete response (CR) or partial response $(\mathrm{PR})$, while 13 patients remained with progressive disease (PD). At the end of follow-up, 11 patients died due to disease progression or other causes.

Correlation between clinical characteristics and OS. The OS of patients in phases I/II (78.9\%) was significantly higher than for those in phases III/IV (20\%, p=0.002, Figure 1A). OS rates of patients that received RT plus CT and CT alone were $76.5 \%$ and $33.3 \%$, respectively $(p=0.012$. Figure $1 B)$. In addition, we found that levels of Ki67 (Figure 1C), $\mathrm{LDH}$ (Figure 1D), ALB (Figure 1E) and ALC (Figure 1F) correlated to OS and the differences of OS were statistically significant between patients with normal clinicopathological characteristics and those with abnormal data (Figure 1). Increased $\mathrm{Ki} 67$ and $\mathrm{LDH}$, as well as decreased ALB and ALC resulted in lower OS in these ENKTL patients.

Gene mutation rates. Among the 29 ENKTL patients, we found mutation rates of $34.48 \%, 31.03 \%, 24.13 \%, 24.13 \%$, $24.13 \%, 17.24 \%, 17.24 \%, 6.89 \%$ and $6.89 \%$ for ARID1A, KMT2D, TP53, MGA, STAT3, EP300, ASXL3, DDX3X and $S T A T 5 B$ genes, respectively (Figure 2). The KMT2D mutation rate was significantly lower in low and low-to-intermediate risk patients than in intermediate-to-high and high risk patients $(18.18 \%$ vs $71.43 \%, \mathrm{p}<0.05$, data not shown) according to their IPI score. However, we found no significant differences in the mutation rates of the other 8 genes between patients with low and high risk.

Associations between KMT2D or TP53 mutation and mortality. Mortality was 75\% (6/8) for patients with KMT2D mutations whereas mortality was $9.5 \%(2 / 21)$ for patients with wild-type $K M T 2 D$, and the difference was statistically significant $(p<0.01)$. Similarly, we found significant 
Table 1. Clinical characteristics of 29 ENKTL patients.

\begin{tabular}{|c|c|c|}
\hline Characteristics & No. & Percentage \\
\hline \multicolumn{3}{|l|}{ Age } \\
\hline$\leq 60$ & 24 & 82.8 \\
\hline$>60$ & 5 & 17.2 \\
\hline \multicolumn{3}{|l|}{ Gender } \\
\hline Male & 24 & 82.8 \\
\hline Female & 5 & 17.2 \\
\hline \multicolumn{3}{|l|}{ Ann Arbor stage } \\
\hline $\mathrm{I} / \mathrm{II}$ & 19 & 65.5 \\
\hline III/IV & 10 & 34.5 \\
\hline \multicolumn{3}{|l|}{ B symptom } \\
\hline With & 20 & 69.0 \\
\hline Without & 9 & 31.0 \\
\hline \multicolumn{3}{|l|}{ Bone marrow involvement } \\
\hline With & 12 & 41.4 \\
\hline Without & 17 & 58.6 \\
\hline \multicolumn{3}{|l|}{ ECOG PS } \\
\hline $0-1$ & 26 & 89.7 \\
\hline$\geq 2$ & 3 & 10.3 \\
\hline \multicolumn{3}{|l|}{ Regional lymph nodes involvement } \\
\hline With & 16 & 55.2 \\
\hline Without & 13 & 44.8 \\
\hline \multicolumn{3}{|l|}{ Extranodal sites } \\
\hline$<2$ & 22 & 75.9 \\
\hline$\geq 2$ & 7 & 24.1 \\
\hline \multicolumn{3}{|l|}{ LDH } \\
\hline$<250 \mathrm{U} / \mathrm{L}$ & 22 & 75.9 \\
\hline$\geq 250 \mathrm{U} / \mathrm{L}$ & 7 & 24.1 \\
\hline \multicolumn{3}{|l|}{ IPI (score) } \\
\hline Low risk $(0-1)$ & 14 & 48.3 \\
\hline Low-to-intermediate risk (2) & 8 & 27.6 \\
\hline Intermediate-to-high risk (3) & 4 & 13.8 \\
\hline High risk (4-5) & 3 & 10.3 \\
\hline \multicolumn{3}{|l|}{ Ki67 } \\
\hline$<70 \%$ & 16 & 55.2 \\
\hline$\geq 70 \%$ & 13 & 44.8 \\
\hline \multicolumn{3}{|l|}{ ALC } \\
\hline$<1.1 \times 10^{9} / \mathrm{L}$ & 13 & 44.8 \\
\hline$\geq 1.1 \times 10^{9} / \mathrm{L}$ & 16 & 55.2 \\
\hline \multicolumn{3}{|l|}{ ALB } \\
\hline$<40 \mathrm{~g} / \mathrm{L}$ & 14 & 48.3 \\
\hline$\geq 40 \mathrm{~g} / \mathrm{L}$ & 15 & 51.7 \\
\hline \multicolumn{3}{|l|}{ Treatment } \\
\hline $\mathrm{CT}+\mathrm{RT}$ & 17 & 58.6 \\
\hline CT & 12 & 41.4 \\
\hline \multicolumn{3}{|l|}{ Treatment effect } \\
\hline $\mathrm{CR}+\mathrm{PR}$ & 16 & 55.2 \\
\hline $\mathrm{PD}$ & 13 & 44.8 \\
\hline
\end{tabular}

ECOG PS, Eastern Cooperative Oncology Group performance status; ALC, absolute lymphocyte count; ALB, albumin; LDH, lactate dehydrogenase; IPI, International Prognostic Index; CT, chemotherapy; RT, radiotherapy; $\mathrm{CR}$, complete response; PR, partial response; PD, progressive disease. Bold fonts denote the clinical parameters for IPI risk stratification. differences in PFS and OS between patients with KMT2D mutations and those with wild-type KMT2D (Figure 3). We also found significant differences in mortality $(\mathrm{p}=0.036)$ between patients with TP53 mutations and wild-type (71.43\% vs $27.27 \%$, data not shown). In order to avoid an imbalanced IPI classification effect, we assessed the difference of mortality between KMT2D mutation and wild-type patients in low and low-to-intermediate risk patients. Statistical results indicated that KMT2D mutations also increased mortality in low and low-to-intermediate risk patients (data not shown). In 22 low and low-to-intermediate risk patients, 3 of 4 patients with KMT2D mutations died whereas only 2 died in 18 patients with wild-type KMT2D ( $\mathrm{p}=0.0058$ ). Although mortality was increased in all patients with TP53 mutations, the difference of mortality was not significant between patient cohorts with TP53 mutation and wild-type in low and low-to-intermediate risk patients.

Integrated System combines KMT2D and TP53 mutations with the IPI score. The difference in PFS was not significant among 4 risk stratifications $(\mathrm{p}=0.0824$, Figure $4 \mathrm{~A}$ ) but OS differed significantly (Figure $4 \mathrm{~B}$ ) according to IPI classification. To devise a more accurate system, we incorporated KMTD2T and TP53 gene mutations into the IPI system as risk factors (a gene mutation is defined as a point). This modification resulted in a redistribution of patients: 13,9 and 7 patients moved to low, intermediate and high risk groups, respectively. The difference in PFS was significant $(\mathrm{p}=0.0094)$ and the new classification showed a homogeneous distribution of patients (Figures 4C and 4D). In order to better assess the improvement of risk stratification integrated with gene mutations, we compared the proportions and OS rates of patients among different risk stratifications between IPI scores and our integrated system (Figure 5). According to IPI classification, the OS rates of low, low-to-intermediate, intermediate-to-high and high risk patients were $78.6 \%$, $62.5 \%, 0 \%$, and $33.3 \%$, respectively. For low-to-intermediate and intermediate-to-high risk groups in the IPI classification, patients with wild-type alleles were reported by our integrated classification to the intermediate risk stratification, while patients with mutations in both genes were resorted to the high risk group. Patients in the intermediate-to-high and high risk groups patients with mutations were reclassified to the high risk group by our integrated system. The OS rates of low, intermediate and high risk patients were $84.6 \%$, $55.6 \%$, and $14.3 \%$ respectively in our integrated system, which formed a harmonious classification with the precise prognosis of ENKTL patients.

\section{Discussion}

ENKTL is also called angiocentric T-cell lymphoma because tumor cells in this disease are derived from natural killer cells and cytotoxic T cells. At present, ENKTL is the most common subtype of $\mathrm{T}$ cell lymphoma in China [16]. However, the outcome of therapeutic interventions for 
Stage

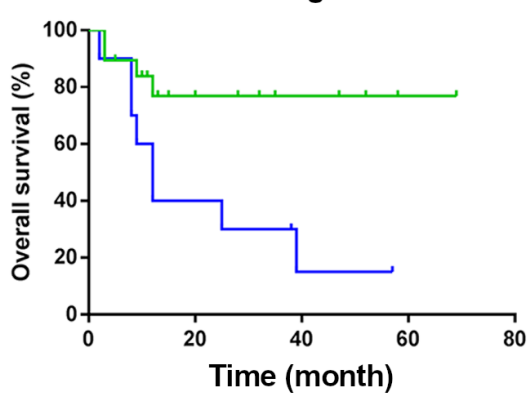

Ki67

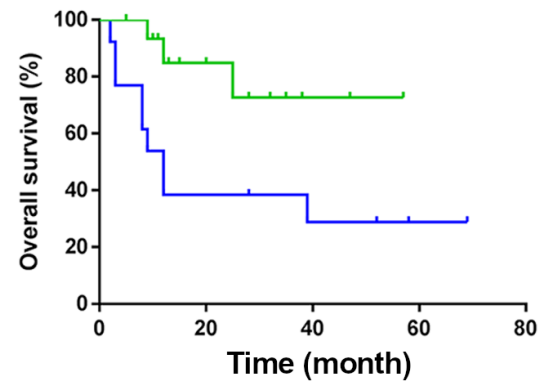

ALB

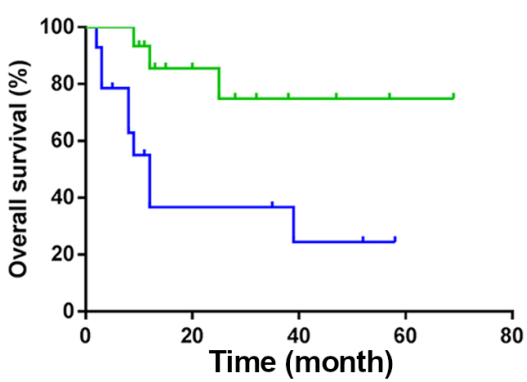

Therapy
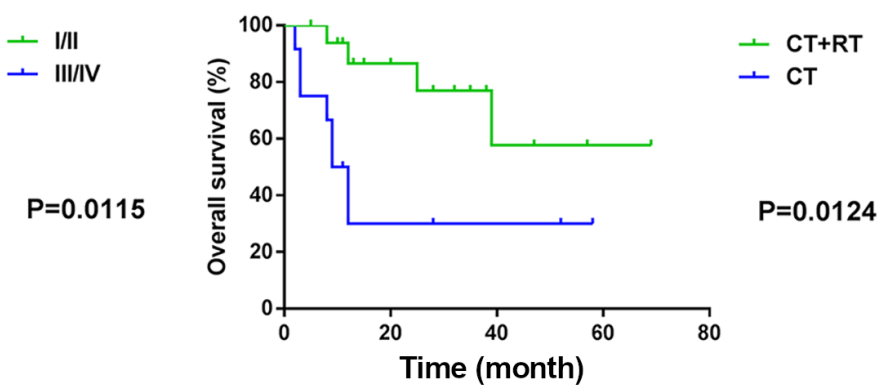

LDH
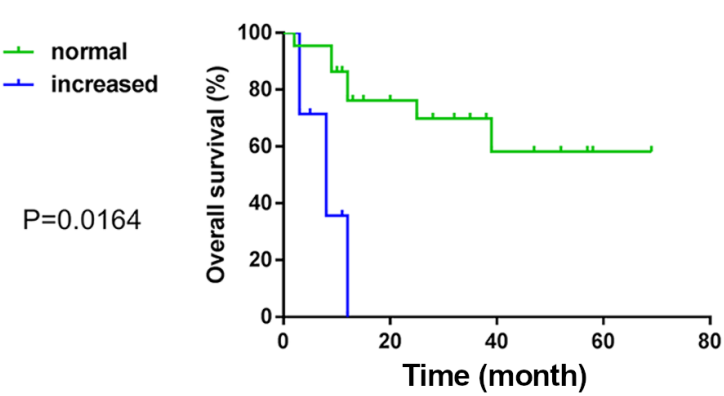

+ normal

- increased

$P=0.0006$

ALC
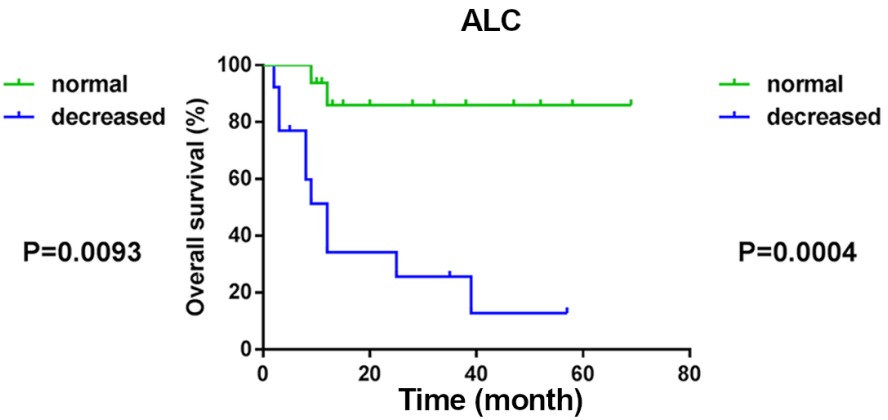

Figure 1. Kaplan-Meier plots of Overall Survival (OS) in patients: comparisons of various clinical characteristics.

ENKTL is generally poor and prognosis varies among patients [17]. Conventional risk stratification for NHL is not specific for ENKTL. In this study, we established a new prognostic model based on the existing IPI system for NHL by incorporating genetic mutation status for a more precise stratification in ENKTL patients.

According to the IPI score designed for NHL, only $24.1 \%$ of 29 ENKTL patients in our cohort were placed in the intermediate-to-high and high risk stratification. This formed an imbalanced classification that did not reflect the high degree of malignancy in ENKTL, suggesting that the IPI score needed improvement to better evaluate ENKTL prognoses. In this study, we found that the status of selected genetic mutations was a powerful factor when incorporated into IPI scores to improve ENKTL patient prognoses.

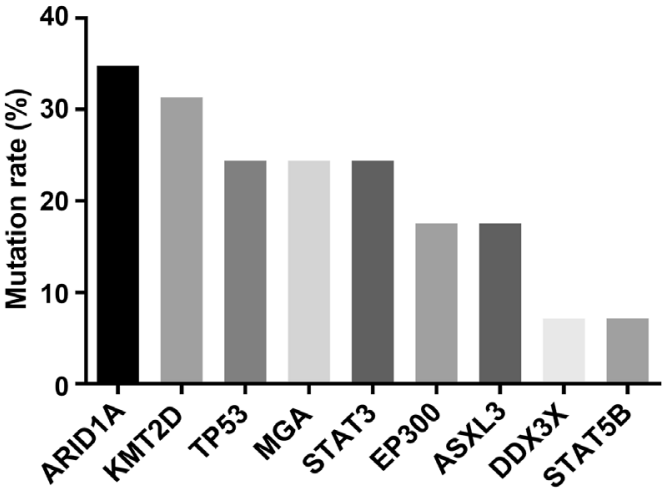

Mutations

Figure 2. Mutation rates of 9 genes evaluated by NGS in 29 patients with ENKTL. 

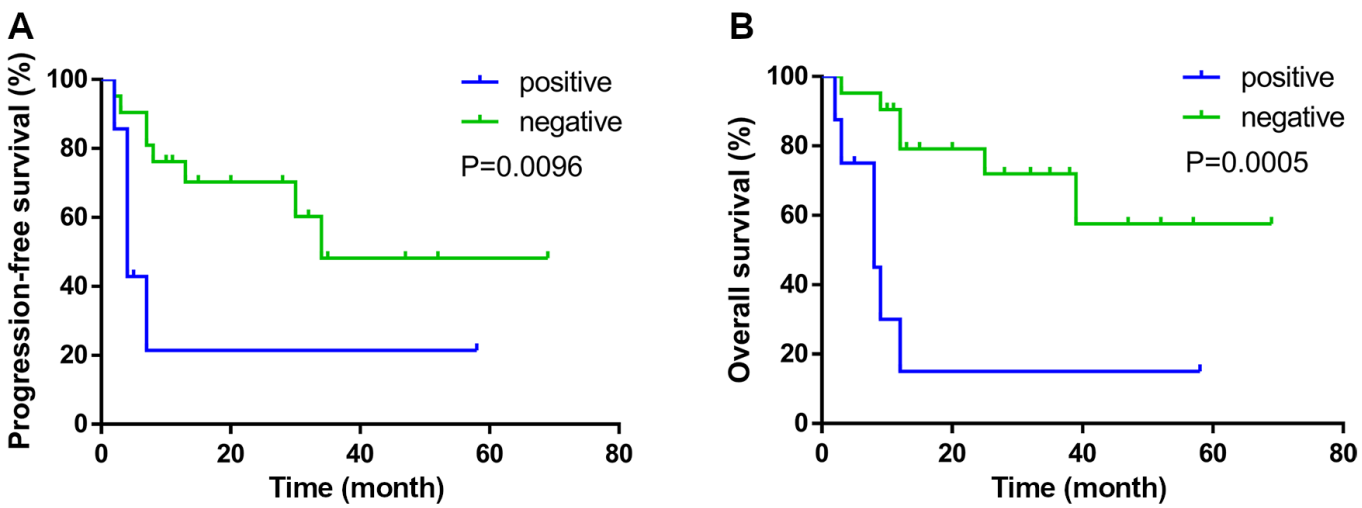

Figure 3. Progression-Free Survival (PFS) and Overall Survival (OS) of patients with mutant and wild-type KMT2D.

A

PFS - IPI classification

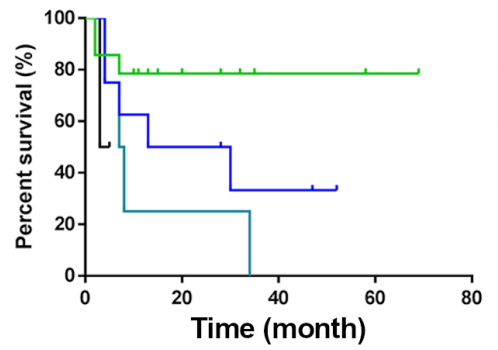

C PFS - integrated classification

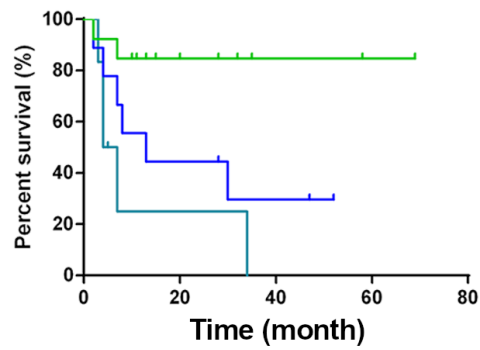

B

OS - IPI classification
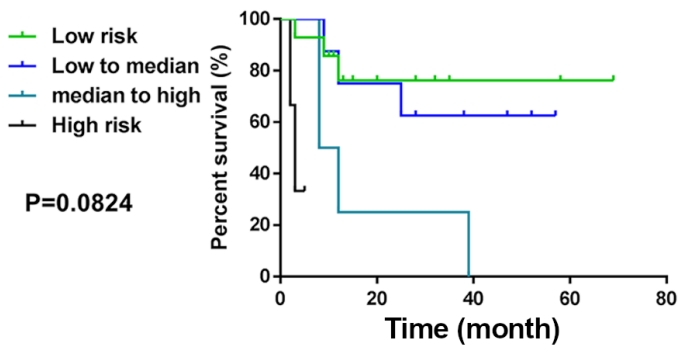

+ Low risk

- Low to median

- median to high

+ High risk

$P=0.0002$
D OS - integrated classification

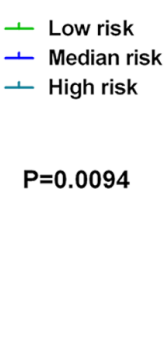

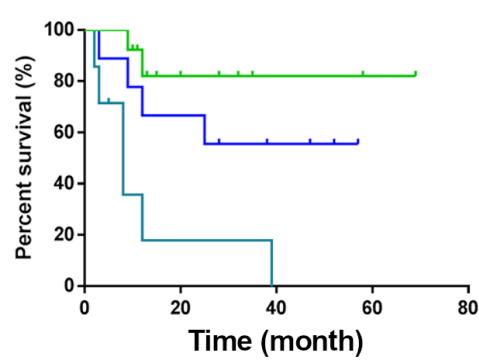

+ Low risk

- Median risk

+ High risk

$P=0.0008$

Figure 4. PFS and OS of ENKTL patients: comparison of risk classification method by stratification category. A) PFS using IPI categories. B) OS using IPI categories. C) PFS using our integrated classification categories. D) OS using our integrated classification categories.

A growing number of studies have shown that numerous genetic mutations are associated with the occurrence and development of ENKTL [18-20]. However, it has been difficult to investigate gene profiles of ENKTL patients because of their low incidence. In recent years, the sensitivity and specificity of NGS techniques have been greatly improved and many gene mutations can be detected simultaneously by a specifically assigned gene panel [21]. In this study, we investigated mutations in a small panel of genes frequently associated with ENKTL, including ARID1A, KMT2D, TP53, MGA, STAT3, EP300, ASXL3, DDX3X, and STAT5B. Our
NGS results showed that except for DDX $3 X$ and STAT5B, the mutation rates of the genes were all over $10 \%$, consistent with a report by Choi et al. [22]. The high mutation rate for KMT2D in intermediate-to-high and high risk patients suggested that mutant KMT2D may more accurately be placed in a high risk stratification and predict poor prognosis. Ardeshir-Larijani et al. [23] reported that patients with mutant KMT2D had a significantly lower median OS and PFS compared with patients with wild-type KMT2D in NSCLC. Consistent with this report, we also found that KMT2D mutant-positive ENKTL patients in our cohort 


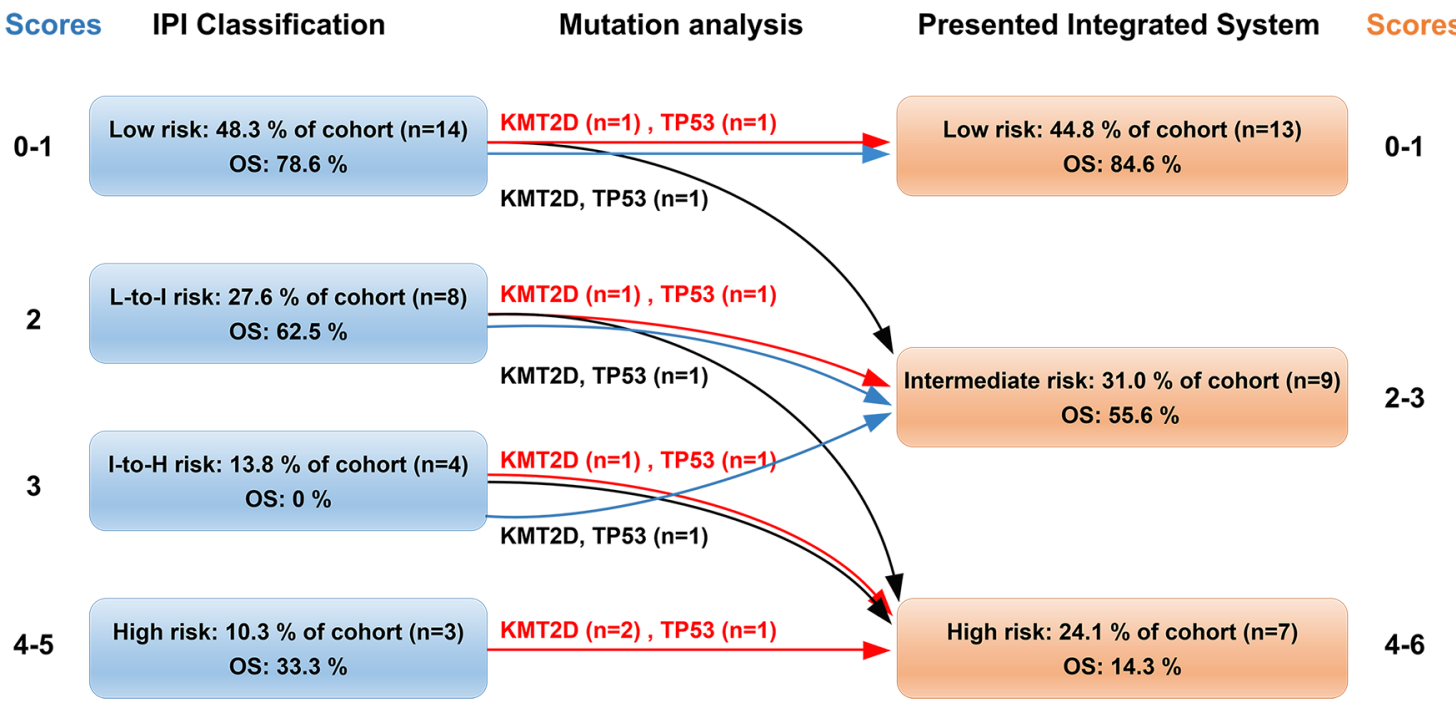

Figure 5. Effect of mutation profiling on conventional IPI risk stratification. Patients with markedly poor prognoses are reassigned into more appropriate risk groups according to their mutation profiles. Red arrows denote patients with either a mutant KMT2D or TP53 gene. Black arrows denote patients with both mutant KMT2D and TP53 genes. Blue arrows denote patients with wild-type genes. L-to-I, low-to-intermediate; I-to-H, intermediate-to-high.

had a lower median OS and PFS. Ortega-Molina et al. [24] showed that KMT2D mutations could promote lymphoma development due to enhanced proliferation and impaired terminal differentiation and KMT2D deficiency was sufficient to trigger $\mathrm{B}$ cell malignancy in mice. Our results showed that KMT2D may be an independent high risk prognostic factor for ENKTL patients. However, the prognostic value of KMT2D mutations in ENKTL needs to be further investigated in a larger cohort of patients. KMT2D (formerly known as MLL2) encodes a catalytic lysine methyltransferase containing SET domain, which has the activity to methylate $\mathrm{H} 3 \mathrm{~K} 4$ and is an important component of the polyprotein complex that plays a role in gene regulation and embryonic development [25-28]. Abnormal expression of KMT2D has been shown to be associated with multiple tumors, such as breast cancer, colon cancer [29], prostate cancer and gastric cancer [30,31]. Several studies reported that KMT2D acted as a tumor suppressor gene in non-Hodgkin lymphoma [32-34]. It was reported that KMT2D deficiency resulted in attenuated cancer cell proliferation and defective cell migration in medulloblastoma cancer [35]. It was reported that KMT2D acted as a tumor suppressor gene, leading to changes in the epigenetic landscape of cancer precursor cells, which promotes the proliferation of mice $\mathrm{B}$ cells. Thus the eradication of KMT2D-deficient cells may be a rational therapeutic strategy for lymphoma [36]. In studies of DLBCL and follicular cell lymphoma, data showed that $89 \%$ of FL and $23-32 \%$ of DLBCL have KMT2D gene mutations, which mostly occur in the early stage of tumor formation. Most of the mutations are nonsense mutations and code shift mutations, which lead to the inactivation of the KMT2D gene [32-34]. Korean researchers also found mutations of the KMT2D gene in ENKTL through the whole exon sequencing technology, counted for about $17.6 \%$, most of which were nonsense mutations. They also found that the mutations caused the loss of gene function through RNA sequencing, suggesting that epigenetic factor mutations may play a role in the pathogenesis of ENKTL [20].

TP53 is a well-known tumor suppressor gene, which encodes a protein P53 to regulate the expression of corresponding target genes in response to various cellular stress, thus inducing regulation of DNA repair, apoptosis, senescence, and DNA repair and metabolism [37, 38]. Majority of TP53 mutations are missense mutations, and the encoded mutant $\mathrm{P} 53$ proteins not only exerts a negative effect on wildtype p53 and inhibits its function as a tumor suppressor [39, 40], but also exhibits new carcinogenic functions including promoting cell proliferation, the evasion of apoptosis, metabolic changes, migration, and so on [41-43]. Clinical studies have demonstrated that mutations in the tumor suppressor gene TP53 predicted poor prognosis in hematopoietic tumors and ENKTL $[10,37,44,45]$. However, in our study TP53 mutation status only predicted increased mortality but had no significant effect on PFS and OS. Additional studies with significantly more patients may be required to establish the predictive value of TP53 mutation status in ENKTL. Nevertheless, for our cohort of ENKTL patients, incorporating the IPI score with KMT2D and TP53 mutation status, produced a new scoring system that could predict a patients' prognosis more precisely.

In conclusion, we identified a new prognostic stratification system by combining the IPI classification for NHL with ENKTL-relevant genetic mutations, resulting in a significant improvement in risk stratification. Additional studies 
with significantly more patients are warranted to validate the clinical relevance of this integrated system for ENKTL, which should provide great insight into disease management of ENKTL and our understanding of ENKTL biology.

Acknowledgements: This work was supported by grants from Science and Technology Project of Hebei Province (No. 13277780D).

\section{References}

[1] STADLMANN S, FEND F, MOSER P, OBRIST P, GREIL $R$ et al. Epstein-Barr virus-associated extranodal NK/Tcell lymphoma, nasal type of the hypopharynx, in a renal allograft recipient: case report and review of literature. Hum Pathol 2001; 32: 1264-1268. https://doi.org/10.1053/ hupa.2001.28962

[2] AU WY, WEISENBURGER DD, INTRAGUMTORNCHAI T, NAKAMURA S, KIM WS et al. Clinical differences between nasal and extranasal natural killer/T-cell lymphoma: a study of 136 cases from the International Peripheral T-Cell Lymphoma Project. Blood 2009; 113: 3931-3937. https://doi. org/10.1182/blood-2008-10-185256

[3] BHATKULE MA, DHAWLE MS, KUMBHAKARNA NR, BINDU RS. Nasal natural killer/T cell lymphoma. Indian J Hematol Blood Transfus 2014; 30: 292-293. https://doi. org/10.1007/s12288-014-0363-7

[4] SUZUKI R. NK/T-cell lymphomas: pathobiology, prognosis and treatment paradigm. Curr Oncol Rep 2012; 14: 395-402. https://doi.org/10.1007/s11912-012-0245-9

[5] LI YJ, YI PY, LI JW, LIU XL, TANG T et al. Prognostic role of $\mathrm{ABO}$ blood type in patients with extranodal natural killer/T cell lymphoma, nasal type: a triple-center study. Chin J Cancer 2017; 36: 62. https://doi.org/10.1186/s40880-017-0229-0

[6] INTERNATIONAL NON-HODGKIN'S LYMPHOMA PROGNOSTIC FACTORS PROJECT. A predictive model for aggressive non-Hodgkin's lymphoma. $\mathrm{N}$ Engl J Med 1993; 329: 987-994. https://doi.org/10.1056/ NEJM199309303291402

[7] SUZUKI R, SUZUMIYA J, YAMAGUCHI M, NAKAMURA S, KAMEOKA J et al. Prognostic factors for mature natural killer (NK) cell neoplasms: aggressive NK cell leukemia and extranodal NK cell lymphoma, nasal type. Ann Oncol 2010; 21: 1032-1040. https://doi.org/10.1093/annonc/mdp418

[8] MCMASTER ML, SUN C, LANDI MT, SAVAGE SA, ROTUNNO M et al. Germline mutations in Protection of Telomeres 1 in two families with Hodgkin lymphoma. Br J Haematol 2018; 181: 372-377. https://doi.org/10.1111/bjh.15203

[9] SCHMITZ R, WRIGHT GW, HUANG DW, JOHNSON CA, PHELAN JD et al. Genetics and Pathogenesis of Diffuse Large B-Cell Lymphoma. N Engl J Med 2018; 378: 13961407. https://doi.org/10.1056/NEJMoa1801445

[10] DOBASHI A, TOGASHI Y, TANAKA N, YOKOYAMA M, TSUYAMA $\mathrm{N}$ et al. TP53 and OSBPL10 alterations in diffuse large B-cell lymphoma: prognostic markers identified via exome analysis of cases with extreme prognosis. Oncotarget 2018; 9: 19555-19568. https://doi.org/10.18632/oncotarget. 24656
[11] PASTORE A, JURINOVIC V, KRIDEL R, HOSTER E, STAIGER AM et al. Integration of gene mutations in risk prognostication for patients receiving first-line immunochemotherapy for follicular lymphoma: a retrospective analysis of a prospective clinical trial and validation in a populationbased registry. Lancet Oncol 2015; 16: 1111-1122. https:// doi.org/10.1016/S1470-2045(15)00169-2

[12] DUNCAVAGE EJ, ABEL HJ, SZANKASI P, KELLEY TW, PFEIFER JD. Targeted next generation sequencing of clinically significant gene mutations and translocations in leukemia. Mod Pathol 2012; 25: 795-804. https://doi.org/10.1038/ modpathol.2012.29

[13] HASLAM K, CATHERWOOD MA, DOBBIN E, SPROUL A, LANGABEER SE et al. Inter-Laboratory Evaluation of a Next-Generation Sequencing Panel for Acute Myeloid Leukemia. Mol Diagn Ther 2016; 20: 457-461. https://doi. org/10.1007/s40291-016-0222-3

[14] CARBONE PP, KAPLAN HS, MUSSHOFF K, SMITHERS DW,TUBIANA M. Report of the Committee on Hodgkin's Disease Staging Classification. Cancer Res 1971; 31: 1860-1861.

[15] Chan JKC, Quintanilla-Martinez L, Ferry JA, Peh SC. Extranodal NK/T-cell lymphoma, nasal type. pp. 285-288. In: SH Swerdlow, E Campo, NL Harris, ES Jaffe, SA Pileri et al. (Eds.). WHO Classification of Tumours of Haematopoietic and Lymphoid Tissues, 4th Edition. IARC, Lyon 2008, p 439. ISBN 978-92-832-2431-0.

[16] CHEN W, ZHENG R, ZENG H, ZHANG S, HE J. Annual report on status of cancer in China, 2011. Chin J Cancer Res 2015; 27: 2-12. https://doi.org/10.3978/j.issn.10009604.2015.01.06

[17] KWONG YL, KIM WS, LIM ST, KIM SJ, TANG T et al. SMILE for natural killer/T-cell lymphoma: analysis of safety and efficacy from the Asia Lymphoma Study Group. Blood 2012; 120: 2973-2980. https://doi.org/10.1182/blood-2012-05-431460

[18] WEN H, MA H, CAI Q, LIN S, LEI X et al. Recurrent ECSIT mutation encoding V140A triggers hyperinflammation and promotes hemophagocytic syndrome in extranodal NK/T cell lymphoma. Nat Med 2018; 24: 154-164. https://doi. org/10.1038/nm.4456

[19] DOBASHI A, TSUYAMA N, ASAKA R, TOGASHI Y, UEDA $\mathrm{K}$ et al. Frequent BCOR aberrations in extranodal NK/T-Cell lymphoma, nasal type. Genes Chromosomes Cancer 2016; 55: 460-471. https://doi.org/10.1002/gcc.22348

[20] LEE S, PARK HY, KANG SY, KIM SJ, HWANG J et al. Genetic alterations of JAK/STAT cascade and histone modification in extranodal NK/T-cell lymphoma nasal type. Oncotarget 2015; 6: 17764-17776. https://doi.org/10.18632/oncotarget.3776

[21] MATA E, DIAZ-LOPEZ A, MARTIN-MORENO AM, SANCHEZ-BEATO M, VARELA I et al. Analysis of the mutational landscape of classic Hodgkin lymphoma identifies disease heterogeneity and potential therapeutic targets. Oncotarget 2017; 8: 111386-111395. https://doi.org/10.18632/oncotarget.22799

[22] CHOI S, GO JH, KIM EK, LEE H, LEE WM et al. Mutational Analysis of Extranodal NK/T-Cell Lymphoma Using Targeted Sequencing with a Comprehensive Cancer Panel. Genomics Inform 2016; 14: 78-84. https://doi.org/10.5808/ GI.2016.14.3.78 
[23] ARDESHIR-LARIJANI F, BHATEJA P, LIPKA MB, SHARMA N, FU P et al. KMT2D Mutation Is Associated With Poor Prognosis in Non-Small-Cell Lung Cancer. Clin Lung Cancer 2018; 19: e489-e501. https://doi.org/10.1016/j. cllc.2018.03.005

[24] ORTEGA-MOLINA A, BOSS IW, CANELA A, PAN H, JIANG Y et al. The histone lysine methyltransferase KMT2D sustains a gene expression program that represses B cell lymphoma development. Nat Med 2015; 21: 1199-1208. https:// doi.org/10.1038/nm.3943

[25] VALLIANATOS CN, IWASE S. Disrupted intricacy of histone H3K4 methylation in neurodevelopmental disorders. Epigenomics 2015; 7: 503-519. https://doi.org/10.2217/epi.15.1

[26] DAWKINS JB, WANG J, MANIATI E, HEWARD JA, KONIALI L et al. Reduced Expression of Histone Methyltransferases KMT2C and KMT2D Correlates with Improved Outcome in Pancreatic Ductal Adenocarcinoma. Cancer Res 2016; 76: 4861-4871. https://doi.org/10.1158/0008-5472. CAN-16-0481

[27] FORD DJ, DINGWALL AK. The cancer COMPASS: navigating the functions of MLL complexes in cancer. Cancer Genet 2015; 208: 178-191. https://doi.org/10.1016/j.cancergen.2015.01.005

[28] JIANG H, SHUKLA A, WANG X, CHEN WY, BERNSTEIN BE et al. Role for Dpy-30 in ES cell-fate specification by regulation of $\mathrm{H} 3 \mathrm{~K} 4$ methylation within bivalent domains. Cell 2011; 144: 513-525. https://doi.org/10.1016/j. cell.2011.01.020

[29] NATARAJAN TG, KALLAKURY BV, SHEEHAN CE, BARTLETT MB, GANESAN N et al. Epigenetic regulator MLL2 shows altered expression in cancer cell lines and tumors from human breast and colon. Cancer Cell Int 2010; 10: 13. https://doi.org/10.1186/1475-2867-10-13

[30] JE EM, LEE SH, YOO NJ, LEE SH. Mutational and expressional analysis of MLL genes in gastric and colorectal cancers with microsatellite instability. Neoplasma 2013; 60: 188-195. https://doi.org/10.4149/neo_2013_025

[31] GRASSO CS, WU YM, ROBINSON DR, CAO X, DHANASEKARAN SM et al. The mutational landscape of lethal castration-resistant prostate cancer. Nature 2012; 487: 239-243. https://doi.org/10.1038/nature11125

[32] MORIN RD, MENDEZ-LAGO M, MUNGALL AJ, GOYA R, MUNGALL KL et al. Frequent mutation of histone-modifying genes in non-Hodgkin lymphoma. Nature 2011; 476: 298-303. https://doi.org/10.1038/nature10351

[33] LOHR JG, STOJANOV P, LAWRENCE MS, AUCLAIR D, CHAPUY B et al. Discovery and prioritization of somatic mutations in diffuse large B-cell lymphoma (DLBCL) by whole-exome sequencing. Proc Natl Acad Sci U S A 2012; 109: 3879-3884. https://doi.org/10.1073/pnas.1121343109
[34] PASQUALUCCI L, TRIFONOV V, FABBRI G, MA J, ROSSI $\mathrm{D}$ et al. Analysis of the coding genome of diffuse large Bcell lymphoma. Nat Genet 2011; 43: 830-837. https://doi. org/10.1038/ng.892

[35] GUO C, CHEN LH, HUANG Y, CHANG CC, WANG P et al. KMT2D maintains neoplastic cell proliferation and global histone H3 lysine 4 monomethylation. Oncotarget 2013; 4: 2144-2153. https://doi.org/10.18632/oncotarget.1555

[36] ZHANG J, DOMINGUEZ-SOLA D, HUSSEIN S, LEE JE, HOLMES AB et al. Disruption of KMT2D perturbs germinal center B cell development and promotes lymphomagenesis. Nat Med 2015; 21: 1190-1198. https://doi.org/10.1038/ nm.3940

[37] JIANG L, GU ZH, YAN ZX, ZHAO X, XIE YY et al. Exome sequencing identifies somatic mutations of DDX3X in natural killer/T-cell lymphoma. Nat Genet 2015; 47: 1061-1066. https://doi.org/10.1038/ng.3358

[38] YIN Y, STEPHEN CW, LUCIANI MG, FAHRAEUS R. p53 Stability and activity is regulated by Mdm2-mediated induction of alternative p53 translation products. Nat Cell Biol 2002; 4: 462-467. https://doi.org/10.1038/ncb801

[39] TERZIAN T, SUH YA, IWAKUMA T, POST SM, NEUMANN $\mathrm{M}$ et al. The inherent instability of mutant p53 is alleviated by Mdm2 or p16INK4a loss. Genes Dev 2008; 22: 1337-1344. https://doi.org/10.1101/gad.1662908

[40] FREBOURG T, KASSEL J, LAM KT, GRYKA MA, BARBIER $N$ et al. Germ-line mutations of the p53 tumor suppressor gene in patients with high risk for cancer inactivate the p53 protein. Proc Natl Acad Sci U S A 1992; 89: 6413-6417. https://doi.org/10.1073/pnas.89.14.6413

[41] BROSH R, ROTTER V. When mutants gain new powers: news from the mutant p53 field. Nat Rev Cancer 2009; 9: 701-713. https://doi.org/10.1038/nrc2693

[42] ZHANG C, LIU J, LIANG Y, WU R, ZHAO Y et al. Tumourassociated mutant p53 drives the Warburg effect. Nat Commun 2013; 4: 2935. https://doi.org/10.1038/ncomms3935

[43] HANEL W, MARCHENKO N, XU S, YU SX, WENG W et al. Two hot spot mutant p53 mouse models display differential gain of function in tumorigenesis. Cell Death Differ 2013; 20: 898-909. https://doi.org/10.1038/cdd.2013.17

[44] GANGAT N, PATNAIK MM, TEFFERI A. Myelodysplastic syndromes: Contemporary review and how we treat. Am J Hematol 2016; 91: 76-89. https://doi.org/10.1002/ ajh.24253

[45] QUINTANILLA-MARTINEZ L, KREMER M, KELLER G, NATHRATH M, GAMBOA-DOMINGUEZ A et al. p53 Mutations in nasal natural killer/T-cell lymphoma from Mexico: association with large cell morphology and advanced disease. Am J Pathol 2001; 159: 2095-2105. https://doi.org/10.1016/ S0002-9440(10)63061-1 